\title{
Metastatic Ureter Urothelial Carcinoma
}

National Cancer Institute

\section{Source}

National Cancer Institute. Metastatic Ureter Urothelial Carcinoma. NCI Thesaurus. Code C150515.

Ureter urothelial carcinoma that has spread to another anatomical site. 